\title{
NORMAL AND QUASINORMAL WEIGHTED COMPOSITION OPERATORS
}

\author{
by JAMES T. CAMPBELL, MARY EMBRY-WARDROP, RICHARD J. \\ FLEMING and S. K. NARAYAN
}

(Received 7 March, 1990)

Introduction. In their paper [1], Campbell and Jamison attempted to give necessary and sufficient conditions for a weighted composition operator on an $L^{2}$ space to be normal, and to be quasinormal. Those conditions, specifically Theorems I and II of that paper, are not valid (see [2] for precise comments on the other results in that paper). In this paper we present a counterexample to those theorems and state and prove characterizations of quasinormality (Theorem 1 below) and normality (Theorem 2 and Corollary 3 below). We also discuss additional examples and information concerning normal weighted composition operators which contribute to the further understanding of this class.

In what follows, $(X, \Sigma, \mu)$ will be a complete $\sigma$-finite measure space. $T: X \rightarrow X$ will be a measurable transformation of $X$ onto itself with the properties that the measure $\mu \circ T^{-1}$ is absolutely continuous with respect to $\mu$, and $\mu \circ T^{-1}$ is finite. We set $h=d \mu \circ T^{-1} / d \mu$. By $T^{-1} \Sigma$ we mean the relative completion of the $\sigma$-algebra generated by $\left\{T^{-1} A: A \in \Sigma\right\}$. With the space $X$ and the measure $\mu$ fixed, if $\Gamma \subseteq \Sigma$ is a $\sigma$-algebra we write $L^{2}(\Gamma)$ as the usual equivalence classes of $\Gamma$ measurable functions whose modulus squared is integrable over $X$.

We denote by $E: L^{2}(\Sigma) \rightarrow L^{2}\left(T^{-1} \Sigma\right)$ the so-called conditional expectation operator with respect to the $\sigma$-algebra $T^{-1} \Sigma$. More generally, $E(f)$ may be defined for bounded measurable functions $f$ or non-negative measurable functions $f$; for details on the properties of $E$ see [1], [3], [4].

Given a $\Sigma$-measurable function $\phi: X \rightarrow \mathbb{C}$, the weighted composition operator (w.c.o.) induced by $T$ with weight $\phi$ is defined by

$$
W_{T, \phi} f(x)=\phi(x) f(T x), \quad f \in L^{2}(\Sigma) .
$$

Usually $\phi$ and $T$ are understood and we just write $W$. The operator norm of $W$ is $\|W\|=\left\|h E\left(|\phi|^{2}\right) \circ T^{-1}\right\|_{\infty}^{1 / 2}$ (see [1] for a discussion of $E(\cdot) \circ T^{-1}$ when $T$ is not invertible). All of our w.c.o.'s will be bounded. The support of a measurable function $g$ is $\bigcup_{n=1}^{\infty}\{x:|g(x)|>1 / n\}$; we shall let supp $g$ denote the support of $g$. Equalities and inequalities between measurable functions are interpreted in the almost everywhere sense, and equality between sets is interpreted up to a set of measure 0 .

We use the following non-standard notation. Whenever $\Gamma$ is a sub- $\sigma$-algebra of $\Sigma$ and $A$ is any $\Sigma$-measurable set, by $\Gamma \cap A$ we mean $\{B \cap A: B \in \Gamma\}$. The statement $\Gamma \cap A=$ $\Sigma \cap A$ means that for each $\Sigma$-measurable set $C \subseteq A$, there exists a set $B \in \Gamma$ so that $B \cap A=C$.

In our statements and proofs of the theorems below, we assume $\phi \geq 0$. The results can be easily extended to the case of a complex-valued $\phi$.

The following example illustrates that the characterizations of normal and quasinormal w.c.o.'s given in [1] are false. 
ExAmple 1. Let $X=\mathbb{Z} \cup\{a\}$ where $a \notin \mathbb{Z}, \Sigma=2^{X}$, and $\mu(x)=1$ for each $x \in X$. Define $T: X \rightarrow X$ by $T(n)=n+1$ for $n \in \mathbb{Z}$, and $T(a)=0$. Define $\phi$ by $\phi(n)=1$ for $n \in \mathbb{Z}$ and $\phi(a)=0$. A straightforward computation shows that $W$ is normal, but that (i) $\phi$ is not $T^{-1} \Sigma$ measurable, (ii) $T^{-1}(\operatorname{supp} \phi) \neq \operatorname{supp} \phi$, and (iii) $h E\left(\phi^{2}\right) \circ T^{-1} \neq h \circ T E\left(\phi^{2}\right)$. Properties (i) and (ii) invalidate Theorem 1 of [1] and property (iii) invalidates Theorem 2 of [1].

The correct characterization of quasinormality is given by the restriction of the condition of Theorem 2 in [1] to the support of $\phi$.

THEOREM 1. W is quasinormal if and only if $h \circ T E\left(\phi^{2}\right)=h E\left(\phi^{2}\right) \circ T^{-1}$ on the support of $\phi$.

Proof. Compute $W W^{*} W f=\phi h \circ T E\left(\phi^{2}\right) f \circ T$ and $W^{*} W W f=h E\left(\phi^{2}\right) \circ T^{-1} \phi f \circ T$. Then $W$ is quasinormal if and only if

$$
\phi h \circ T E\left(\phi^{2}\right) f \circ T=h E\left(\phi^{2}\right) \circ T^{-1} \phi f \circ T, \text { for all } f \in L^{2} .
$$

This last condition is equivalent to the one in the statement of the theorem.

REMARK. Theorem 1 may be proved using the polar decomposition approach attempted in [1] provided one observes a factor of $\phi$ in each term of $V M$ and $M V$, where $M=\left|W^{*} W\right|^{1 / 2}$ and $V$ is the partial isometry which gives the unique, canonical polar form $V M=W$.

We may now establish the correct characterization of normality in many ways, and the most useful would be one which required the fewest and easiest calculations involving $h, \phi, E$, and $T$. The following theorem gives a minimal set of conditions which are necessary and sufficient for the normality of $W$. Since supp $\phi \in \Sigma$, by our previous convention condition (ii) in the following theorem means that for each $B \in \Sigma, B \subseteq \operatorname{supp} \phi$, there exists $C \in \Sigma$ so that $T^{-1} C \cap \operatorname{supp} \phi=B$.

THEOREM 2. W is normal if and only if

(i) $\phi E(\phi) h \circ T=h E\left(\phi^{2}\right) \circ T^{-1}$, and

(ii) $T^{-1} \Sigma \cap \operatorname{supp} \phi=\Sigma \cap \operatorname{supp} \phi$.

Proof. Suppose (i) and (ii) are true. Since $h \circ T>0$, it follows from (i) that $\operatorname{supp} h E\left(\phi^{2}\right) \circ T^{-1}=\operatorname{supp} \phi E(\phi)$. Moreover for any non-negative $f$ we have supp $f E(f)=$ supp $f$, so that $\operatorname{supp} h E\left(\phi^{2}\right) \circ T^{-1}=\operatorname{supp} \phi$. Now let $B$ be a $T^{-1} \Sigma$-measurable set with finite measure. From (i) and the support condition just established,

$$
\phi E\left(\phi \chi_{B}\right) h \circ T=\phi E(\phi) h \circ T \chi_{B}=h E\left(\phi^{2}\right) \circ T^{-1} \chi_{B},
$$

where $\chi_{B}$ denotes the indicator function of $B$. From (ii) it follows that (1) holds for any $\Sigma$-measurable subset $B$ of supp $\phi$, as long as $B$ has finite measure. Again, since $\operatorname{supp} \phi=\operatorname{supp} h E\left(\phi^{2}\right) \circ T^{-1}$, (1) holds for all $\Sigma$-measurable sets $B$ of finite measure. Consequently $W W^{*} \chi_{B}=W^{*} W \chi_{B}$ for all such $B$, implying that $W$ is normal.

Assume that $W$ is normal. Then $W W^{*} f=W^{*} W f$ for all $f \in L^{2}(\Sigma)$. This is equivalent to

$$
\phi h \circ T E(\phi f)=h E\left(\phi^{2}\right) \circ T^{-1} f, \text { for all } f \in L^{2}(\Sigma),
$$

which implies that

$$
\phi h \circ T E(\phi) f=h E\left(\phi^{2}\right) \circ T^{-1} f, \quad \text { for all } f \in L^{2}\left(T^{-1} \Sigma\right) .
$$


From (2)' it follows easily that (i) is true. Combining (i) and equation (2) we have

$$
\phi E(\phi f)=\phi E(\phi) f, \quad f \in L^{2}(\Sigma) .
$$

We now consider the special case where $\phi>0$. Then by (3) $E(\phi f)=E(\phi) f$ for all $f \in L^{2}(\Sigma)$. In particular for $f=\chi_{B}$ with $\mu(B)$ finite we obtain

$$
\int_{X} \phi \chi_{B} d \mu=\int_{X} E\left(\phi \chi_{B}\right) d \mu=\int_{X} E(\phi) \chi_{B} d \mu .
$$

Therefore

$$
\int_{B} \phi d \mu=\int_{B} E \phi d \mu \text { for all } B \in \Sigma, \quad \mu(B)<\infty .
$$

Consequently $E(\phi)=\phi$. It now follows from equation (3) that $E(f)=f$ for all $L^{2}$ functions $f$, or equivalently that $T^{-1} \Sigma=\Sigma$.

We now drop the restriction that $\phi>0$. Since $W$ is normal, $\overline{\operatorname{ran} W}$ (the closure of the range of $W$ ) is a reducing subspace for $W$. By (3) we have that $W W^{*} f=\phi h \circ T E(\phi f)=$ $\phi h \circ T E(\phi) f$, so that $\overline{\operatorname{ran} W}=L^{2}(\operatorname{supp} \phi E(\phi), \Sigma, \mu)=L^{2}(\operatorname{supp} \phi, \Sigma, \mu)$. Thus $L^{2}(\operatorname{supp} \phi, \Sigma, \mu)$ is a reducing subspace for $W$ on which $W$ is normal. On the other hand $\operatorname{ran} W=\operatorname{ran} W^{*}=L^{2}\left(\operatorname{supp} h E\left(\phi^{2}\right) \circ T^{-1}\right)$, so that $\operatorname{supp} \phi=\operatorname{supp} h E\left(\phi^{2}\right) \circ T^{-1}$. Consequently $\phi(T x)=0$ implies that $h(T x) E\left(\phi^{2}\right)(x)=0$ which implies that $\phi(x)=0$. Therefore $T$ maps the support of $\phi$ into itself, and we see that $W$ is a normal w.c.o. on $L^{2}(\operatorname{supp} \phi, \Sigma, \mu)$. For this space the weight is non-zero, so if we define $\Sigma_{1}:=\Sigma \cap$ supp $\phi$ and $T_{1}$ as the restriction of $T$ to supp $\phi$, the preceding paragraph implies that $T_{1}^{-1} \Sigma_{1}=\Sigma_{1}$, which is precisely condition (ii).

REMARK 1. In the preceding proof it is shown that if $\phi E(\phi) h \circ T=h E\left(\phi^{2}\right) \circ T^{-1}$ (in particular if $W$ is normal), then supp $\phi=\operatorname{supp} h E\left(\phi^{2}\right) \circ T^{-1}$. Moreover, if $\operatorname{supp} h E\left(\phi^{2}\right) \circ$ $T^{-1} \subseteq \operatorname{supp} \phi$, then $T$ maps supp $\phi$ into itself so that $W$ is a weighted composition operator on the invariant subspace $L^{2}(\operatorname{supp} \phi, \Sigma, \mu)$.

Remark 2. If $\phi>0$, then, by Theorem $1, W$ is quasinormal if and only if $h \circ T E\left(\phi^{2}\right)=h E\left(\phi^{2}\right) \circ T^{-1}$ and, by Theorem $2, W$ is normal if and only if $T^{-1} \Sigma=\Sigma$ and $\phi^{2} h \circ T=h \phi^{2} \circ T^{-1}$. In particular if $\phi \equiv 1, W$ is quasinormal exactly when $h \circ T=h$ and normal exactly when $h \circ T=h$ and $T^{-1} \Sigma=\Sigma$. These last two results are found in [5] and are two of the earliest results connecting operator theoretic properties of $W$ and measure-theoretic properties of $T$.

In [4] Lambert proves that $W$ is hyponormal if and only if (i) $\operatorname{supp} \phi \subseteq \operatorname{supp} h E\left(\phi^{2}\right) \circ$ $T^{-1}$, and (ii) $h \circ T E\left(\frac{\phi^{2}}{h E\left(\phi^{2}\right) \circ T^{-1}}\right) \leq 1$ (where the fraction is interpreted as 0 off of $\left.\operatorname{supp} h E\left(\phi^{2}\right) \circ T^{-1}\right)$. It is reasonable to conjecture that equality in (ii) will imply normality. However equality holds in the following example in which $W$ is not even quasinormal.

EXAMPLE 2. Let $\phi \equiv 1, X=\{n\}_{n=0}^{\infty} \cup\left\{a_{k}\right\}_{k=1}^{\infty} \cup\left\{b_{k}\right\}_{k=1}^{\infty}, \Sigma$ the $\sigma$-algebra of all subsets of $X$. Define $T: X \rightarrow X$ by $T\left(a_{k+1}\right)=a_{k}$ and $T\left(b_{k+1}\right)=b_{k}$ for $k \geq 1, T\left(a_{1}\right)=$ $T\left(b_{1}\right)=0$, and $T(n)=n+1$ for all $n \geq 0$. Define the measure by $\mu\left(a_{k}\right)=2^{k-1}$ and 
$\mu\left(b_{k}\right)=2^{2 k-1}$ for $k \geq 1$, and $\mu(n)=3^{-n}$ for $n \geq 0$. Direct computation shows that $h \circ T E\left(\frac{\phi^{2}}{h E\left(\phi^{2}\right) \circ T^{-1}}\right)=1$ but that $h\left(a_{1}\right) \neq h\left(T a_{1}\right)$ so that $W$ is not quasinormal.

We can, however, generalize Lambert's result to the normal case, and the following corollary gives conditions for normality analogous to his conditions for hyponormality.

COROLlaRY 3. $W$ is normal if and only if the following conditions hold.

(i) $\operatorname{supp} \phi=\operatorname{supp} h E\left(\phi^{2}\right) \circ T^{-1}$,

and

(ii) $T^{-1} \Sigma \cap \operatorname{supp} \phi=\Sigma \cap \operatorname{supp} \phi$,

(iii) $h \circ T E\left(\frac{\phi^{2}}{h E\left(\phi^{2}\right) \circ T^{-1}}\right)=\chi_{\text {supp } E(\phi)}$,

where the fraction is interpreted to be 0 off of $\operatorname{supp} h E\left(\phi^{2}\right) \circ T^{-1}$.

Proof. Assume that $W$ is normal so that (i) and (ii) are true by Remark 1 and Theorem 2. Therefore

$$
h \circ T E\left(\frac{\phi^{2}}{h E\left(\phi^{2}\right) \circ T^{-1}}\right)=h \circ T E\left(\frac{\phi^{2}}{\phi E(\phi) h \circ T}\right)=E\left(\frac{\phi}{E(\phi)} \chi_{\text {supp } E(\phi)}\right),
$$

where the last equality follows since $\operatorname{supp} \phi \subseteq \operatorname{supp} E(\phi)$. Finally this last expression equals $\chi_{\text {supp } E(\phi)}$.

Now assume that (i), (ii), and (iii) are true. By Theorem 2 we need only show that $\phi E(\phi) h \circ T=h E\left(\phi^{2}\right) \circ T^{-1}$. It follows from (iii) that

$$
E\left(\phi \frac{\phi E(\phi) h \circ T}{h E\left(\phi^{2}\right) \circ T^{-1}} f \circ T\right)=E(\phi f \circ T), \text { for all } f \in L^{2}(\Sigma),
$$

or $E(k \phi f \circ T)=0$ for all $f \in L^{2}(\Sigma)$, where $k=\frac{\phi E(\phi) h \circ T}{h E\left(\phi^{2}\right) \circ T^{-1}}-1$. In particular, if $C \in \Sigma$ has finite measure and $f=\chi_{C}$ we may conclude that

$$
\int_{T^{-1}(C) \text { nsupp } \phi} k \phi \mathrm{d} \mu=0
$$

Condition (ii) now implies that $k \phi=0$. This, together with condition (i), implies that $\phi E(\phi) h \circ T=h E\left(\phi^{2}\right) \circ T^{-1}$, and the conditions of Theorem 2 are satisfied.

It is an open question whether conditions analogous to those in Corollary 3 exist in the quasinormal case.

AcKnowledgement. We thank Prof. Alan Lambert of the University of North Carolina at Charlotte for his helpful and informative communications concerning this paper.

\section{REFERENCES}

1. J. Campbell and J. Jamison, On some classes of weighted composition operators, Glasgow Math. J. 32 (1990), 87-94. 
2. J. Campbell and J. Jamison, Errata to: On some classes of weighted composition operators, Glasgow Math. J. 32 (1990), 261-263.

3. S. Foguel, The ergodic theory of Markov processes, Math. Studies No. 21 (Van Nostrand Reinhold, New York, 1969).

4. A. Lambert, Hyponormal composition operators, Bull. London Math. Soc. 18 (1986), 395-400.

5. R. Whitley, Normal and quasinormal composition operators, Proc. Amer. Math. Soc. 70 (1978), 114-118.

James T. Campbell:

Department of Mathematical Sciences

Memphis State University

MEMPhIS, TN. 38152

U.S.A.
Other Authors:

Department of Mathematics

Central Michigan University

Mt. Pleasant, Michigan 48859

U.S.A. 